\title{
ANÁLISE SOBRE A INFLUÊNCIA DO MÉTODO DE AMOSTRAGEM DE SÓLIDOS EM TRANSPORTADOR PNEUMÁTICO EM CIRCUITO FECHADO
}

\author{
V. M. B. MATSUMOTO, T. F. PÁDUA* \\ Universidade Federal de São Carlos, Departamento de Engenharia Química \\ e-mail: tfpadua@gmail.com
}

\begin{abstract}
RESUMO
O transportador pneumático de sólidos é importante devido à grande quantidade e diversidade de materiais que é capaz de transportar. Além disso, muitas operações industriais utilizam transportadores para processamento, como ocorre na secagem de sólidos por transporte (flash dryer). Os estudos em transportadores pneumáticos precisam vir acompanhados de medidas confiáveis de vazão de sólidos. O objetivo deste trabalho foi o de analisar a influência de dois diferentes métodos de amostragem de partículas sobre o valor da vazão mássica de sólidos calculada para o transporte pneumático. Experimentos foram realizados introduzindo partículas esféricas de vidro (com cerca de $1 \mathrm{~mm}$ de diâmetro) em um transportador pneumático com a configuração de leito fluidizado circulante e foram usados dois reservatórios distintos para recolher o material sólido. Ambos tinham as mesmas dimensões e eram feitos do mesmo material, mas um possuía escape para o ar proveniente do transportador e o outro não. $\mathrm{O}$ escape era constituído por um recorte no galão que foi devidamente coberto por uma tela, a fim de evitar que os sólidos fossem arrastados para fora. $\mathrm{O}$ reservatório com escape para $\mathrm{o}$ ar mostrou-se mais apropriado para amostragem da vazão de sólidos no caso do equipamento em questão, que não possuía exaustão de ar antes da caixa de amostragem de sólidos. Além disso, foi proposto um novo método para medir a vazão de sólidos nesse tipo de sistema para evitar desvios sistemáticos durante a amostragem.
\end{abstract}

\section{INTRODUÇÃO}

As partículas sólidas com variados diâmetros são de grande interesse aos processos químicos devido ao grande número de aplicações na engenharia (YANG, 2003), como o uso na catálise heterogênea. Além disso, grande parte dos produtos e subprodutos industriais se encontra no estado sólido.

Frequentemente o material sólido precisa ser transportado entre diferentes pontos do processo industrial ou para estocagem e distribuição ao mercado. Marcus et al. (1990) destacaram que a alternativa preferencial para esse papel é o transporte pneumático de sólidos, em que partículas são transportadas por uma corrente de gás, comumente ar.

Além do papel mencionado, muitos sistemas de transporte pneumático podem ser utilizados no processamento de material sólido, se beneficiando da grande área de contato entre as partículas e a fase fluída, como ocorre na secagem em transportador.

Uma configuração comum de operação do transportador é a de leito fluidizado circulante (CFB). Em um transportador deste tipo as partículas sólidas são repostas continuamente ao circuito. Esta configuração 
é comum no craqueamento catalítico de petróleo (FCC), mas aplicações extensivas em secagem ainda não são comuns (GRACE, AVIDAN \& KNOWLTON, 1997). A secagem a partir de um transportador deste tipo ainda está sendo estudada e sua viabilidade avaliada (SOUZA et al., 2010; RAJAN et al. 2006; RAJAN et al. 2008).

Várias configurações de secador CFB foram propostas (SOUZA et al., 2010; RAJAN et al. 2006; LOPES et al. 2011). Entre elas, a alimentação feita no trecho ascendente, ao reduzir uma curva no transportador, tende a diminuir a perda de carga, o atrito e a degradação dos sólidos. Assim, alternativas desse tipo parecem ser preferíveis e uma dessas alternativas foi utilizada nesse trabalho, com um dispositivo Venturi em orientação vertical, cuja função é a de evitar o desvio da corrente de ar para o duto de alimentação (MILLS, 2004).

O objetivo deste trabalho foi a verificação da influência do método de amostragem sobre a vazão mássica medida no transportador. A mensuração adequada desta variável é fundamental para a caracterização do transporte pneumático, o que trás importância ao presente estudo.

A medida da vazão de sólidos não é facilmente obtida nesses sistemas. Medidas indiretas são complexas e ainda pouco generalizadas (MILLS, 2004; CROWE et al., 2012), diferente do que ocorre com o escoamento de uma só fase (DELMÉE, 2003). A medida direta pode ser realizada em estudos no transportador pneumático através do desvio dos sólidos ao final do transportador (PATROCÍNIO, 2006).

A amostragem foi feita com o desvio dos sólidos por uma caixa de amostragem. Porém, avaliou-se se a possibilidade de escape de ar no recipiente de coleta de sólidos influenciava nas medidas de vazão. Para isto, foram usados dois galões distintos para recolher o material sólido desviado. Ambos tinham o mesmo tamanho e eram feitos do mesmo material, mas um possuía escape para $\mathrm{o}$ ar proveniente do transportador e o outro não.

O transportador utilizado não possuía escape para o ar na região anterior à caixa de amostragem de sólidos para permitir o estudo do trecho descendente em toda a sua extensão. Assim, o transportador não incluía um ciclone. Com os resultados apresentados, é possível demonstrar que nesse caso é indispensável utilizar escape para o ar no reservatório de coleta.

\section{MATERIAIS E MÉTODOS}

A Figura 1 mostra o transportador pneumático circulante utilizado. Um soprador de 7,5HP (ERBELE, São Paulo, Brasil) com capacidade de $380 \mathrm{~m}^{3} / \mathrm{h}$ fez o suprimento de ar no sistema pelo conduto horizontal.

Figura 1 - Transportador pneumático circulante utilizado.

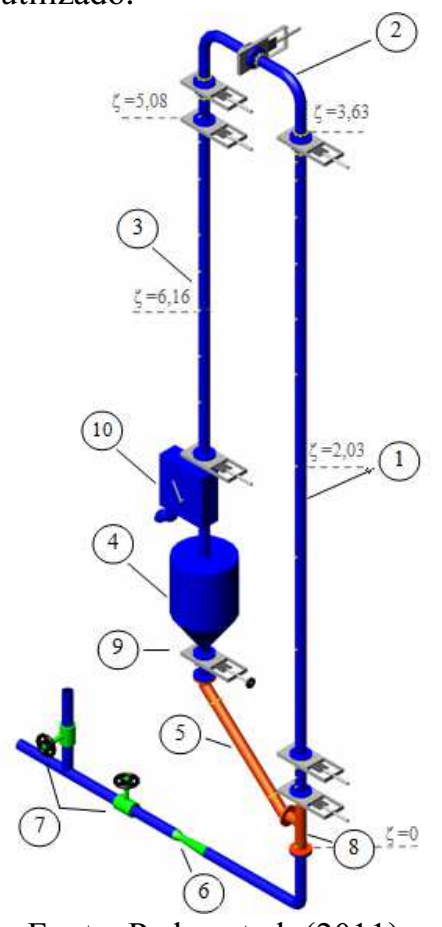

Fonte: Padua et al. (2011)

Uma válvula globo restringia a passagem de ar diretamente na tubulação 
horizontal de alimentação. Um dispositivo Venturi encontrava-se logo em seguida e estava conectado ao manômetro de água para medição da vazão volumétrica de ar no sistema. Antes que as esferas de vidro de $1 \mathrm{~mm}$ de diâmetro fossem inseridas no transportador para os ensaios com material particulado, o equipamento passou por uma fase de estabilização que durava cerca de uma hora.

Após completar o transportador pneumático circulante, as partículas chegavam a uma caixa de amostragem que poderia conduzi-las até um silo armazenador onde o material sólido permanecia até ser reposto à corrente de transporte - ou poderia retirá-las do sistema, numa situação de amostragem. A vazão mássica foi obtida através da massa de partículas sólidas colhidas na amostragem e o tempo que se levou para que tal massa fosse retirada. A caixa de amostragem opera através de uma alavanca, como mostra a Figura 2.

Figura 2 - Caixa de amostragem.

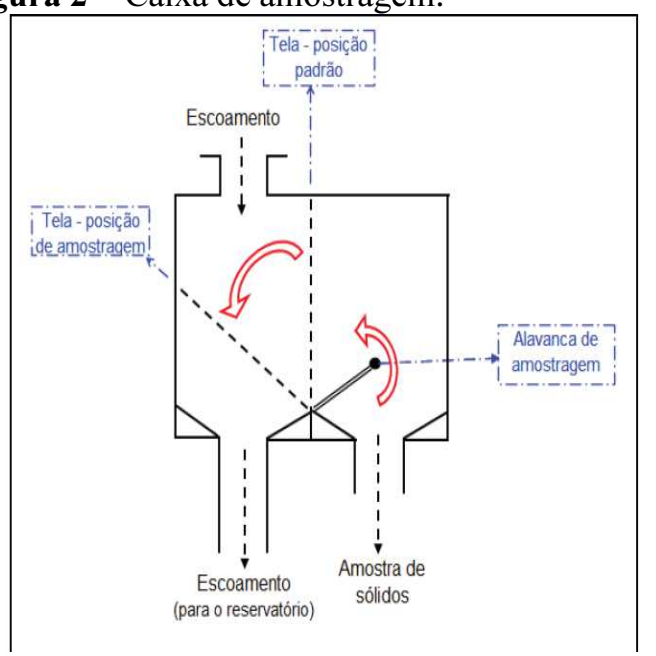

Fonte: Pádua (2012)

Enquanto a amostragem não era realizada, o escoamento levava ao reservatório de sólidos do transportador (lado esquerdo da Figura 2) ou silo de armazenamento de sólidos do transportador, de formato cônico em sua base e com capacidade para armazenar cerca de $50 \mathrm{~kg}$ de sólidos. Uma válvula guilhotina na saída do silo servia como meio de controle para a vazão de sólidos. Na conexão entre o conduto de transporte e o conduto lateral (segmento que ligava o silo ao conduto de transporte) havia um dispositivo Venturi projetado para sistemas à baixa pressão. $\mathrm{O}$ mesmo alimentador foi utilizado por Pádua, 2012.

A amostragem desviava os sólidos com a utilização de uma tela interna à caixa de amostragem (desvio para o lado direito da Figura 2). Os sólidos desviados eram levados por uma mangueira flexível até o reservatório de coleta (ou reservatório de amostragem) de particulado. Foram utilizados dois reservatórios distintos de coleta de amostras, apresentados na Figura 3, que se tratavam de galões vazios de água de $5 \mathrm{~L}$.

As partículas retiradas durante a amostragem foram colhidas primeiramente por um galão (reservatório) todo fechado, mostrado na Figura 3(a), para todas as vazões volumétricas utilizadas. Posteriormente, a amostragem foi feita, também para todas as vazões volumétricas, utilizando-se um galão semelhante ao primeiro, que se distinguia apenas pela presença de um recorte em sua estrutura devidamente coberto por uma tela (reservatório com escape para o ar), mostrado na Figura 3(b). $O$ procedimento de amostragem foi seguido igualmente para os dois galões, de maneira que apenas a vazão volumétrica e o recipiente que colhia as amostras foram alterados durante $o$ experimento a fim de que as influências dos mesmos sobre a amostragem pudessem ser observadas.

O material sólido foi coletado pelo menos três vezes em cada vazão volumétrica utilizada. As partículas eram constantemente repostas através da caixa de amostragem, que possui contato com o exterior, de maneira a evitar grande alteração na altura do leito no silo. 
Figura 3 - Reservatórios de amostragem. a - galão sem escape para o ar. b - galão com escape para o ar (reservatório de coleta com tela).

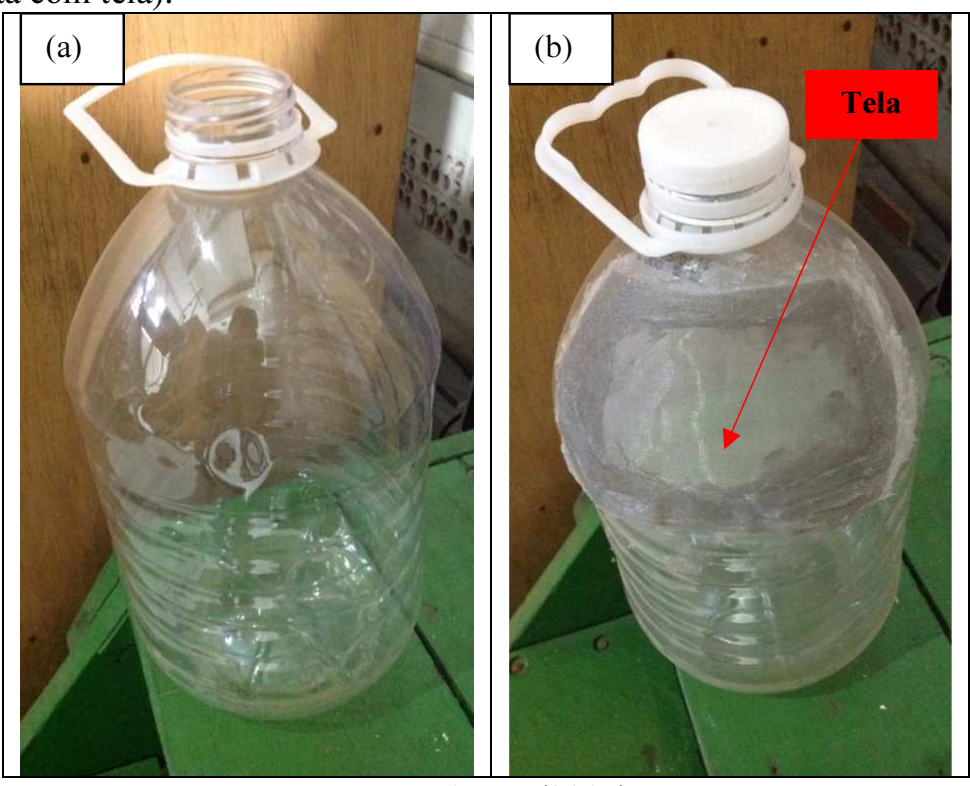

Fonte: Autor (2015)

Após cada alteração na vazão de ar, trinta minutos se passavam até que o sistema se estabilizasse novamente e então as medidas eram efetuadas.

O tempo de amostragem não foi escolhido arbitrariamente. Padua (2012) observou experimentalmente que o tempo de amostragem necessário até que a vazão mássica se estabilizasse seria por volta de 30 segundos. Assim, foram realizadas amostragens entre 15 e $60 \mathrm{~s}$, de modo à avaliar a variação das amostras com o tempo. Foi utilizada uma balança Filizola MF-I de erro igual a $0,02 \mathrm{~kg}$ para a pesagem das amostras de sólidos recolhidas e para controle de tempo utilizou-se um cronômetro.

\section{RESULTADOS E DISCUSSÕES}

\subsection{Resultados da amostragem com diferentes reservatórios}

As discrepâncias das vazões obtidas com o uso dos dois diferentes galões podem ser observadas através dos gráficos de vazão mássica medida em função do tempo de coleta de sólidos (Figura 4) e pelas vazões mássicas para cada vazão volumétrica com ambos os galões (Tabela 1) utilizando o tempo nominal de 35 segundos de amostragem. Os resultados apresentados na Figura 4 acompanham o erro padrão para a vazão (medidas em triplicata).

A Figura 5 mostra as massas recolhidas em função do tempo de coleta da amostra para a vazão e $255,07 \mathrm{~m}^{3} / \mathrm{h}$. Assim, é possível perceber que a curva para o galão sem tela parece se estabilizar a partir de 30s. Essa não é uma característica boa de amostragem, pois a massa coletada sempre deve aumentar com o tempo de amostragem. O comportamento linear obtido para o reservatório com escape para o ar é mais apropriado e representa um valor de vazão mais estável em relação ao tempo de amostragem. 
Figura 4 - Vazão mássica em função do tempo de amostragem para cada galão na vazão volumétrica de $255,07 \mathrm{~m}^{3} / \mathrm{h}$. a reservatório sem tela. $\mathrm{b}$ - reservatório com tela.
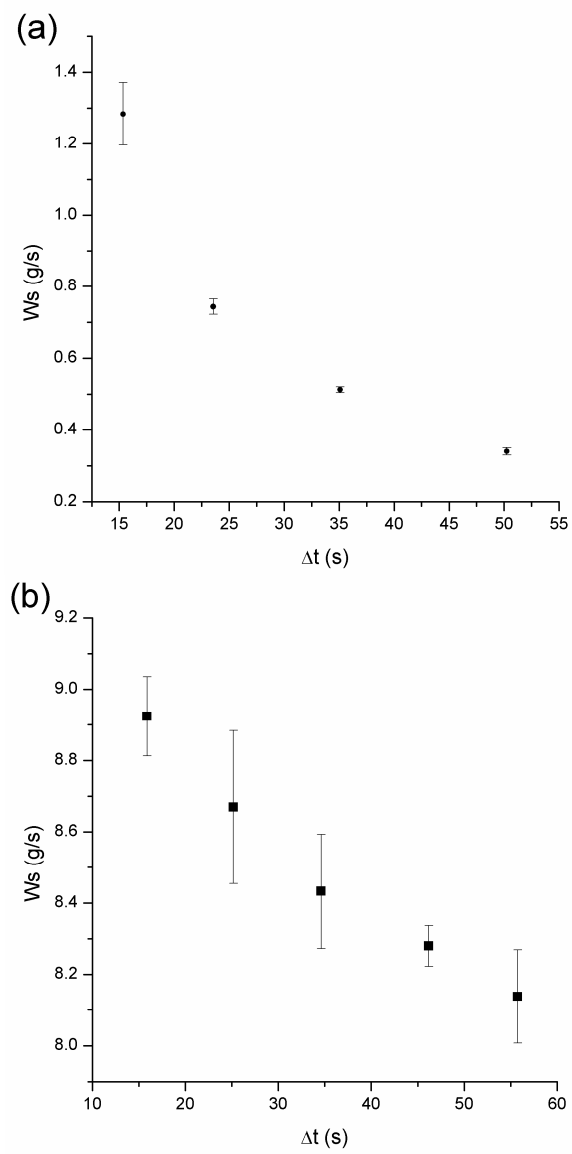

Fonte: Autor (2015)

Tabela 1 - Vazões mássicas para cada vazão volumétrica aplicada para ambos os galões utilizados. $35 \mathrm{~s}$ nominais de amostragem.

\begin{tabular}{|c||c|c|}
\hline $\begin{array}{c}\text { Q } \\
\left(\mathrm{m}^{3} / \mathrm{h}\right)\end{array}$ & $\begin{array}{l}\text { Ws - galão com } \\
\text { tela }(\mathrm{g} / \mathrm{s})\end{array}$ & $\begin{array}{l}\text { Ws - galão sem } \\
\text { tela }(\mathrm{g} / \mathrm{s})\end{array}$ \\
\hline 107,5 & 6,65 & 2,76 \\
\hline 165,7 & 7,20 & 1,12 \\
\hline 225,1 & 7,48 & 0,51 \\
\hline 255,1 & 8,28 & 0,51 \\
\hline 274,5 & 9,25 & 0,47 \\
\hline
\end{tabular}

Ademais, o erro associado ao valor obtido para um determinado tempo de amostragem em relação ao tempo anterior para o galão sem tela atinge a casa dos $30 \%$, mesmo em tempos longos de amostragem, enquanto que para o outro galão está abaixo de $5 \%$, mesmo que utilizando somente 25 segundos de amostragem. Tais dados são melhor representados pelos gráficos da Figura 6.

Figura 5 - Massa recolhida em função do tempo de amostragem para a vazão volumétrica de $255,07 \mathrm{~m}^{3} / \mathrm{h}$. a - reservatório sem tela. $b$ - reservatório com tela.
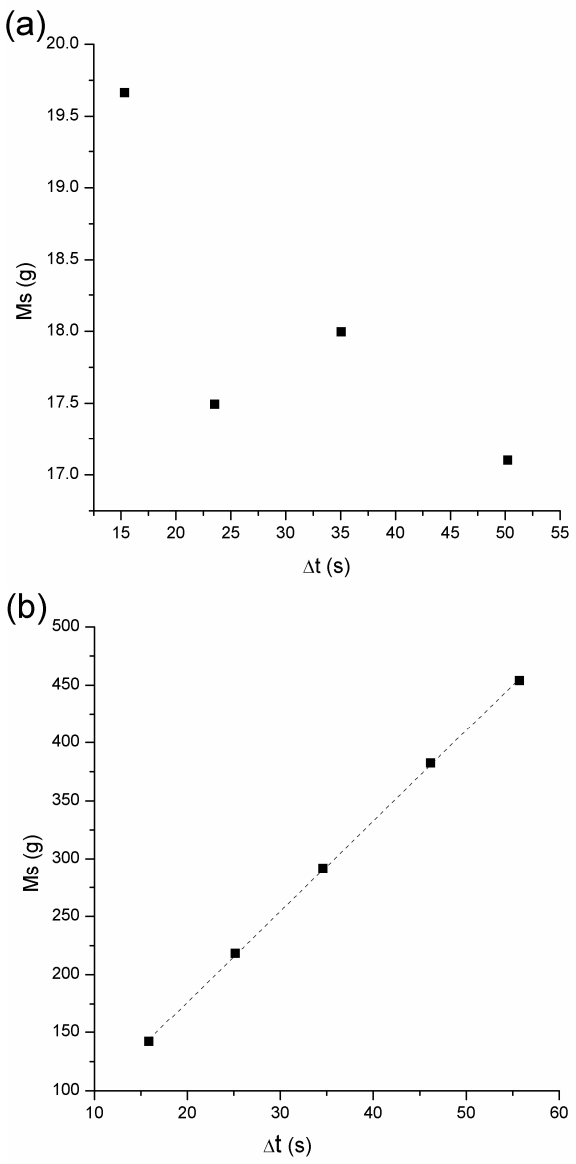

Fonte: Autor (2015)

Pode-se notar que o reservatório com escape para o ar é capaz de fornecer pouco menos de $3 \%$ de variação na medida com coleta de mais de 30 s de amostragem. Mais de 40s forneceram variação em torno de $1,8 \%$. Nesse caso, pelo menos $25 \mathrm{~s}$ de coleta são 
recomendados, de maneira que o reservatório que possui escape para o ar estabiliza a vazão mássica medida em tempos de coleta menores, favorecendo as medidas experimentais.

Além da mais rápida estabilização do reservatório com escape, o reservatório sem tela mostrou a inconsistência destacada na Figura 5, com relação à estabilização da massa coletada. Assim, é mais apropriado utilizar o reservatório com escape para o ar.

Figura 6 - Desvio associado a uma medição de vazão volumétrica a um certo tempo de amostragem em relação ao tempo anterior para a vazão de $255,07 \mathrm{~m}^{3} / \mathrm{h}$. . a - reservatório sem tela. $b$ - reservatório com tela..

(a)
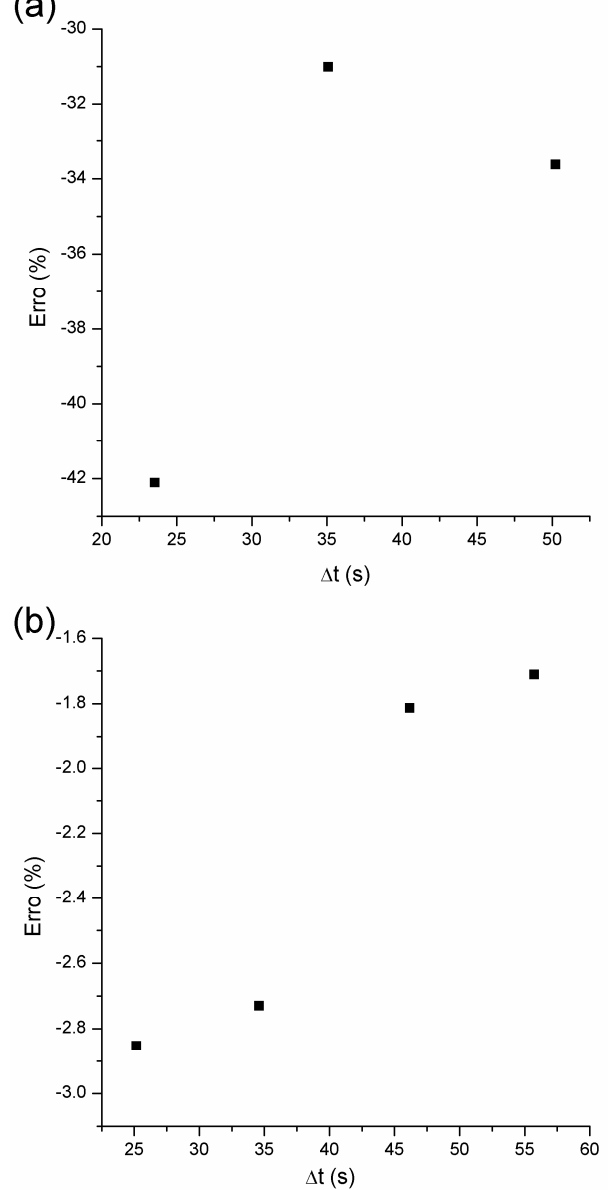

Fonte: Autor (2015).

\subsection{Comparação com resultados anteriores}

Para o galão sem tela, as vazões mássicas calculadas diminuíam conforme a vazão volumétrica aumentava (Tabela 1), o que contrariava a lógica de que elas deveriam ser diretamente proporcionais, bem como foi concluído por Padua (2012). Já o galão com tela atendia a tais expectativas.

A Figura 5 indica que a massa coletada durante a amostragem do reservatório sem a tela estabilizava com o tempo de amostragem. Portanto, a vazão calculada era cada vez menor com a passagem do tempo, o que foi destacado na Figura 4. Esse comportamento é comum aos vários níveis de vazão de ar utilizados.

Os resultados obtidos a partir do galão com tela aproximam-se dos resultados obtidos por Padua (2012) com o mesmo transportador e utilizando também um reservatório com escape para o ar, embora seu coeficiente determinação $\left(\mathrm{R}^{2}\right)$ tenha sido mais satisfatório (0.989 contra 0.825). Ambos apresentaram uma curva crescente e a variação da vazão de sólidos está na casa dos $30 \%$ quando se passa da menor vazão volumétrica para a maior, como pode ser observado na Figura 7. As barras de erro indicam uma probalidade de $95 \%$.

Figura 7 - Vazão mássica em função da vazão volumétrica para o galão com tela. Comparação com resultados de Pádua (2012).

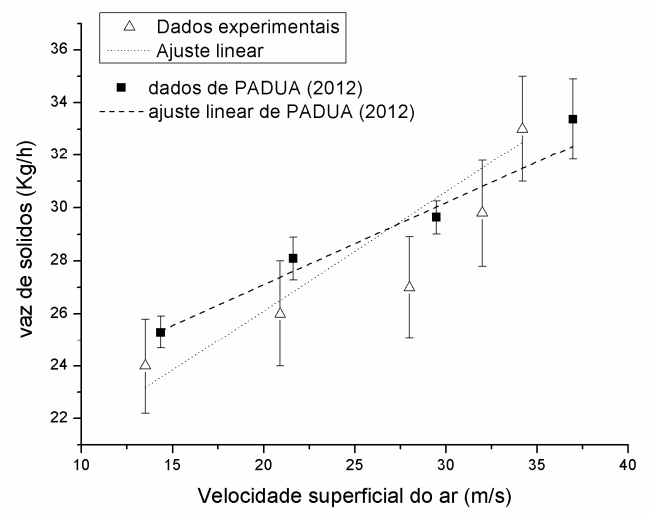

Fonte: Autor (2015). 
A caixa de amostragem de sólidos foi reformada entre os resultados de Padua (2012) e os obtidos no trabalho atual. As telas foram trocadas, assim como a vedação entre a parede da caixa de amostragem e o aparato usado para desviar os sólidos. Sendo essa a única distinção e mantidas as condições operacionais (inclusive a abertura da válvula guilhotina de liberação de sólidos).

Idealmente, o método de medida não pode oferecer desvios sistemáticos e não houve evidencias de que isso ocorreu no caso da caixa de amostragem usada. A diferença obtida nas retas ajustadas somente destaca a natureza do modelo empirico utilizado (linear).

\subsection{Análise das discrepâncias entre os dois métodos de coleta}

A estabilização da massa coletada pôde ser observada experimentalmente ao se utilizar o reservatório sem escape para o ar, já que o galão era transparente. Quando a alavanca era colocada em posição para amostragem de sólidos, uma considerável quantidade de partículas se depositava no galão fechado e a passagem de sólidos era drasticamente reduzida após alguns segundos. Nas maiores vazões, ela praticamente cessava.

A explicação para tal comportamento se encontra na ação da pressão dentro do reservatório no momento da amostragem. Idealmente, o ar deveria seguir pela tela da caixa de amostragem e somente os sólidos seguiriam para o reservatório de coleta.

Em uma situação real de amostragem, parte do ar que vem do transportador era conduzido pelo tubo conectado ao galão. Este reservatório permitia a saída do ar que acompanhava o material particulado somente pelo espaço entre a mangueira de coleta e a parede do reservatório. Assim, ocorria aumento da perda de carga associada ao trecho final do transportador quando amostrando sólidos em reservatório fechado.
Verificada pelo aumento da pressão anterior à caixa durante a amostragem.

O aumento de pressão mencionado tinha dois efeitos que ajudam a explicar as observações experimentais com o reservatório de coleta sem escape de ar.

Em primeiro lugar, o aumento de pressão reduzia a vazão de ar para o sistema durante a amostragem, o que reduzia a vazão de sólidos (Figura 7). Porém, apesar do galão sem tela incluir maior perturbação na vazão de ar (Q) do que o galão com tela, não foi observada variação considerável da vazão medida pelo dispositivo Venturi do trecho horizontal quando a alavanca era alterada para a posição de amostragem, conforme indica a Tabela 2 para três ensaios em condição de vazão distinta. Assim, a redução de vazão não é justificativa suficiente para explicar o comportamento do sistema com o reservatório sem tela de escape.

Tabela 2 - Comportamento da vazão de ar no sistema para três ensaios em níveis distintos de vazão para as condições sem amostragem e com amostragem utilizando os diferentes reservatórios.

\begin{tabular}{|l|r|}
\cline { 2 - 2 } \multicolumn{1}{c|}{} & $\mathrm{Q}\left(\mathrm{m}^{3} / \mathrm{h}\right)$ \\
\hline \hline Sem amostrar & 113,7 \\
\hline Amostragem com tela & 110,9 \\
\hline Amostragem sem tela & 113,7 \\
\hline \hline Sem amostrar & 237,9 \\
\hline Amostragem com tela & 233,8 \\
\hline Amostragem sem tela & 237,2 \\
\hline Sem amostrar & 269,5 \\
\hline Amostragem com tela & 266,5 \\
\hline Amostragem sem tela & 270,1 \\
\hline
\end{tabular}

Em segundo lugar, o aumento da pressão no final do trecho descendente do transportador promovia maior desvio da corrente de ar de transporte pelo conduto lateral. A razão para isto era o aumento de perda de carga no sistema circulante, que fazia com que a corrente de ar tendesse a seguir em maior quantidade para o conduto de alimentação ao invés de seguir pelo sistema, 
uma vez que o reservatório de sólidos encontrava-se em pressão atmosférica.

O desvio da corrente de ar pode ter grande impacto na vazão alimentada de sólidos (COSTA, CARMO \& FREIRE, 2004). Lopes et al. (2011) mostrou que esse desvio pode ser considerável até mesmo com a presença de bocais restritivos. Neste caso, o desvio do ar reduz ou até mesmo impede a corrente de sólidos de ser inserida no sistema (COSTA, CARMO \& FREIRE, 2004; LOPES et al., 2011).

A comprovação de que o reservatório fechado pode ampliar o desvio da corrente de ar de transporte, reduzindo consideravelmente a quantidade de sólidos transportada pôde ser testada ao realizar medidas experimentais da queda de pressão no conduto lateral, do mesmo modo que foi utilizado por Costa, Carmo \& Freire, 2004 e Lopes et al., 2011. Assim, é possível estimar a vazão desviada através da equação de Forchheimer, ao considerar que no conduto de alimentação o material sólido se comporta como um leito poroso sendo percolado por ar.

A Tabela 3 sintetiza os resultados de queda de pressão e vazão volumétrica do ar de transporte desviada pelo conduto lateral. As medidas foram realizadas em três casos: operação sem realizar a amostragem, amostragem utilizando reservatório sem tela e reservatório com tela.

Apesar da porcentagem de ar desviada ser relativamente pequena durante a operação do transportador, o valor é em muito ampliado durante a amostragem de sólidos quando se utiliza o reservatório sem tela. Por exemplo, no caso da maior vazão de transporte utilizada, houve aumento de $125 \%$ em relação à operação do transportador sem amostragem. $\mathrm{O}$ aumento permanece na ordem de $100 \%$, mesmo na menor vazão utilizada.

Este aumento explica o comportamento de coleta de sólidos durante a utilização de reservatório fechado. Por esta razão, o uso de um reservatório sem escape para o ar altera de modo considerável a vazão de sólidos que percorre o transportador, o que leva a medidas incorretas.

Os dados destacaram também que mesmo o reservatório com tela promove variação da vazão de ar desviada. A variação é menor e se reduz à medida que a vazão de ar é reduzida no transportador, sendo desprezível para o menor nível de vazão de ar utilizada. Assim, o reservatório que garante escape para o ar de transporte é mais apropriado. Porém, ainda assim pode incluir desvios em relação à operação do transportador (sem a amostragem). Neste caso, a amostragem reduzia a corrente de ar desviada pelo conduto de alimentação, uma vez que reduzia a perda de carga no transportador durante a amostragem.

Os resultados apresentados servem de alerta que alterações relevantes na perda de carga do sistema devido à amostragem podem ocasionar desvios sistemáticos relevantes nas medidas de vazão mássica usando caixa de amostragem, se o sistema estiver utilizando um alimentador não mecânico.

Tabela 3 - Queda de pressão e vazão desviada pelo conduto de alimentação de sólidos em diferentes vazões.

\begin{tabular}{|l|c|c||c|c||c|c|}
\cline { 2 - 7 } \multicolumn{1}{c|}{} & \multicolumn{5}{c|}{ Vazão de transporte: } \\
\cline { 2 - 7 } \multicolumn{1}{c|}{} & \multicolumn{2}{c|}{$255,1 \mathrm{~m}^{3} / \mathrm{h}$} & \multicolumn{2}{c|}{$237,9 \mathrm{~m}^{3} / \mathrm{h}$} & \multicolumn{1}{c|}{$107,5 \mathrm{~m}^{3} / \mathrm{h}$} \\
\cline { 2 - 7 } \multicolumn{1}{c|}{} & $\Delta \mathrm{P} / \mathrm{L}$ & $\mathrm{Q}_{\text {desv }}$ & $\Delta \mathrm{P} / \mathrm{L}$ & $\mathrm{Q}_{\text {desv }}$ & $\Delta \mathrm{P} / \mathrm{L}$ & $\mathrm{Q}_{\text {desv }}$ \\
\cline { 2 - 7 } & $\mathrm{Pa} / \mathrm{m}$ & $\mathrm{m}^{3} / \mathrm{h}$ & $\mathrm{Pa} / \mathrm{m}$ & $\mathrm{m}^{3} / \mathrm{h}$ & $\mathrm{Pa} / \mathrm{m}$ & $\mathrm{m}^{3} / \mathrm{h}$ \\
\hline Sem amostrar & $-890,0$ & 0.28 & -756.5 & 0.27 & -400.5 & 0.14 \\
\hline Amostrando sem tela & $-2091,6$ & 0.72 & -1691.1 & 0.59 & -801.0 & 0.29 \\
\hline Amostrando com tela & $-534,0$ & 0.19 & -578.5 & 0.21 & -400.5 & 0.14 \\
\hline
\end{tabular}


Vale destacar que o transportador utilizado não possuía escape para o ar na região anterior à caixa de amostragem. Casos que possuam um ciclone antes da caixa de amostragem devem reduzir ou eliminar o efeito negativo de se utilizar o reservatório sem escape para o ar durante a coleta de sólidos para a amostragem.

No caso do sistema utilizado neste trabalho, a construção de um reservatório com escape para o ar, que possuísse uma tela de retenção seguida de uma válvula regulável automática na exaustão poderia garantir menos desvios em relação à operação do transportador. Assim, a perda de pressão no conduto de alimentação poderia ser ajustada durante a amostragem para se aproximar daquela obtida durante a operação. Entretanto, o uso somente seria prático se fosse utilizado um sistema instrumentado (válvula automática e transdutor de pressão).

\section{CONCLUSÕES}

Para as mesmas quantidades de sólidos e vazão volumétrica, os galões com e sem escape de ar apresentaram resultados bastante discrepantes, o que comprova a influência dos meios de amostragem sobre os cálculos. Além disso, o tempo de amostragem influi no valor da vazão mássica calculado e deve-se esperar um período de tempo suficiente para que a relação entre quantidade de sólidos coletados e tempo transcorrido se estabilize.

$O$ galão sem tela deixa de coletar quantidades apreciáveis de sólido depois do início da amostragem, o que foi confirmado visualmente. Assim, os resultados servem de alerta para que se utilizem reservatórios com escape para o ar, ao menos em casos cuja amostragem seja realizada anterior à secção de escape de ar do transportador (sem ciclone anterior à amostragem).

Foi comprovado que o reservatório sem tela é inapropriado por promover maior desvio da corrente de ar de transporte durante a amostragem. Qualquer sistema que altere a distribuição de perda de carga no transportador circulante pode ocasionar desvios sistemáticos na vazão mensurada, pela mesma razão avaliada para o reservatório sem tela.

O reservatório com tela mostrou-se mais apropriado, levando a menores variações na vazão de ar desviada. Ainda assim, frente às alterações de vazão desviada indicadas, foi proposto um novo método de medida de vazão de sólidos para casos similares ao avaliado neste trabalho: utilizar uma válvula na exaustão do reservatório para manter a queda de pressão no conduto de alimentação durante a amostragem.

\section{NOMENCLATURA}

$$
\begin{array}{ll}
\text { Ws } & \text { vazão mássica de sólidos } \\
\text { Ms } & \text { massa coletada de sólidos } \\
\Delta \mathrm{t} & \text { tempo de amostragem (coleta) } \\
\mathrm{Q} & \text { vazão volumétrica de ar } \\
\mathrm{Q}_{\text {desv }} & \begin{array}{l}
\text { vazão de ar desviada para o } \\
\text { conduto de alimentação }
\end{array}
\end{array}
$$

\section{REFERÊNCIAS}

COSTA I A; CARMO M F; FREIRE J T. Analysis of regime transitionsandflow instabilities in a vertical conveying of coarse particles using different solids feeding systems. The Canadian Journal Of Chemical Engineering, v.82, p.48-59, 2004.

CROWE C.T; SCHWARZKOPF J.D; SOMMERFELD M; TSUJI Y. Multiphase flows with droplets and particles. 2012. CRC Press. p. 494.

DELMÉE G. J. Manual de medição de vazão. 2003. Edgard Blucher, terceira edição. 
GRACE J R; AVIDAN A A, KNOWLTON T M. (editores). Circulating fluidized beds. 1997. Blackie Academic \& Professional, Chapman and Hall, Londres.

LOPES C S. Estudo da alimentação de sólidos em sistemas de transporte pneumático. 2011. Tese de doutorado, programa de pós-graduação em engenharia química, UFSCar, São Carlos, 2011.

MARCUS, R. D.; LEUNG, L. S.; KLINZING, G. E.; RISK, F. Pneumatic conveying of solids. 1990. 575p. Londres: Chapman and Hall.

MILLS D. Pneumatic conveying design guide. 2004. Elsevier, segunda edição, 637 p..

PÁDUA T.F. Simulação de um alimentador Venturi em trecho ascendente no transporte pneumático de sólidos. 2012. Tese de doutorado, programa de pósgraduação da UFSCar, São Carlos.

PÁDUA T.F.; LOPES C.S; FREIRE J.T. Estudo experimental sobre a alimentação de sólidos em dispositivos Venturi de orientação vertical para transportadores pneumáticos de material particulado. XXXV Congresso Brasileiro de Sistemas Particulados, 2011, Vassouras - RJ. Anais... Vassouras, UFRRJ, 1992. p.10.

PATROCÍNIO A B. Contribuição ao estudo do escoamento gás-sólido na presença de curvas. 2006. Tese de doutorado, programa de pós-graduação em engenharia química, UFSCar, São Carlos.

RAJAN, K. S.; SRIVASTAVA, S. N.; PITCHUMANI, B.; MOHANTY, B. Simulation of gas-solid heat transfer during pneumatic conveying: Use of multiple gas inlets along the duct. Int. Comm. Heat Mass Transfer, 33, 1234-1242, 2006.
RAJAN, K. S.; DHASANDHAN, K.; SRIVASTAVA, S. N.; PITCHUMANI B. Studies on gas-solid heat transfer during pneumatic conveying. Int. J. Heat Mass Transfer, 51, 2801-2813, 2008

SOUSA, R. C.; ALMEIDA, A. R. F.; FERREIRA, M. C.; FREIRE, J. T. Analysis of fluid dynamics and thermal behavior using a vertical conveyor with a spouted bed feeder. Drying Technol, 28, 1277-1287, 2010

YANG W. Handbook of fluidization and fluid-particle systems. 2003. CRC Press, p.1868.

\section{AGRADECIMENTOS}

Os autores agradecem à CAPES e ao $\mathrm{CNPq}$ pelo apoio financeiro. 